\title{
Length of Vascularized Fibula Graft Utilized and Union: Is There a Relationship?
}

\author{
Jacob Didesch, MD ${ }^{1}$ Virak Tan, MD ${ }^{1}$ \\ 1 Department of Orthopaedics, Rutgers-New Jersey Medical School, \\ Newark, New Jersey \\ J Reconstr Microsurg Open 2016;1:19-25.
}

\begin{abstract}
Address for correspondence Virak Tan, MD, Institute for Hand \& Arm Surgery, 33 Bleeker Street, Suite 103, Millburn, NJ 07041

(e-mail: tanvi@njms.rutgers.edu).
\end{abstract}

\begin{abstract}
Keywords

- nonunion

- bone defects

- vascularized fibula graft

Background Vascularized fibula grafting is used in the treatment of large skeletal defects, recalcitrant atrophic nonunions, and infected nonunions. Few studies have examined the factors contributing to nonunion of the graft at one or both graftrecipient junctions. The purpose of this study was to determine if a correlation exists between length of the graft and primary union of the graft-recipient interface at the proximal and/or distal junction.

Methods A total of 21 patients who underwent vascularized fibula grafting from 2002 to 2014 for the treatment of skeletal defects were included. Radiographs were assessed for union of the graft at the proximal and distal junctions. The rates of union at the proximal and distal junctions were determined with respect to the limb and graft. The relationship between the length of graft utilized and union was assessed.

Results A total of $71.4 \%$ of patients, with an average follow-up of 30.2 months, achieved complete union at an average of 8.7 months. With respect to the limb, a union rate of $95.2 \%$ was achieved at the distal graft-recipient junction versus $71.4 \%$ at the proximal junction $(p=0.038)$. With respect to the graft orientation, a union rate of $90.5 \%$ was achieved at the distal graft versus $76.2 \%$ at the proximal graft $(p=0.214)$. The length of the graft did not have a significant correlation to the rate of union.

Conclusion The length of the fibula graft does not have an association with the rate of primary union. If primary union is not achieved, likely the nonunion will have occurred at the proximal graft-recipient junction.
\end{abstract}

Since the introduction of vascularized fibula grafting using microvascular techniques by Taylor et $\mathrm{al}^{1}$ in 1975 , this technique has been used to treat several difficult conditions. Large skeletal defects greater than 5 to $6 \mathrm{~cm}$ resulting from trauma or resection of pathologic tissue (tumors, congenital pseudoarthrosis, osteomyelitis) have been treated successfully with vascularized fibula grafts. ${ }^{2,3}$ Similarly, this technique has proven successful in achieving union in the treatment of nonunions, including recalcitrant atrophic nonunions and infected nonunions. ${ }^{4-6}$

Union rates for vascularized fibula grafts for the abovementioned conditions have been reported in the literature. In one study, primary union was achieved in $84 \%$ of patients who underwent grafting for traumatic bone defect, nonunion, excision of tumor, excision of osteomyelitis, kyphoscoliosis, and congenital pseudoarthrosis. ${ }^{3}$ A literature review of vascularized free fibula grafts in the treatment of nonunion of long bones reported primary union was achieved in $71.5 \% .^{5}$ Overall, there is a fairly high rate of primary union. Furthermore, secondary union has been achieved with a second procedure in 15 to $20 \%$ of cases in some series. ${ }^{2,5}$

This still leaves a significant number of patients that do not achieve union with the use of vascularized fibula grafting or require a subsequent operation. Few studies have looked directly at the factors that contribute to nonunion of the graft at one or both ends, and specifically at the relationship of received

April 28, 2015

accepted after revision

November 28, 2015

published online

February 15, 2016
DOI http://dx.doi.org/

10.1055/s-0036-1571294. ISSN 2377-0813.
Copyright $\odot 2016$ by Thieme Medical Publishers, Inc., 333 Seventh Avenue, New York, NY 10001, USA. Tel: +1(212) 584-4662.
License terms

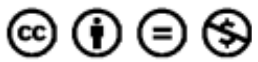


length of the graft to rate of primary union, secondary union, or nonunion. The purpose of this study was to determine if a correlation exists between length of the vascularized fibula graft and primary union of the graft-recipient interface at the proximal and/or distal junction.

\section{Materials and Methods}

After approval from the institutional review board, the charts of 27 consecutive patients who underwent vascularized fibula grafting from 2002 to 2014 were reviewed. Patients were included if they underwent vascularized fibula grafting for the treatment of posttraumatic bone defects, infected nonunions, osteomyelitis, reconstruction following tumor resection, nonunions secondary to tumor reconstructions, and posttraumatic nonunions. Patients were excluded if they underwent the procedure for treatment of hip avascular necrosis (AVN) or congenital pseudoarthrosis, or did not have postoperative follow-up X-rays.

Of the 27 patients who underwent vascularized fibula grafting, one was excluded due to indication of AVN of the hip, one was excluded for nonunion of femoral neck secondary to a gunshot wound, and three were excluded for inadequate or no postoperative follow-up X-rays (graft lengths 15,5 , and 8 $\mathrm{cm}$ ). One patient, with a graft length of $7 \mathrm{~cm}$, was not included because the patient was readmitted to the hospital 2.5 weeks post-vascularized fibula grafting to the radius for resection of renal cell metastasis with respiratory failure from metastasis to the lungs and discharged to hospice. This left a total of 21 patients, 13 males and 8 females, with an average age of 31.5 years (7-72 years) (-Tables $\mathbf{1}$ and $\mathbf{2}$ ). The average length of follow-up was 30.2 months (range, 5-91 months).

From the operative reports, the indication for surgery, operative technique, length of the fibula graft used, and orientation of the graft in the defect were obtained. Orientation of the graft was determined based on the flow of the blood supply to the graft and was recorded as being placed

Table 1 Patient demographics

\begin{tabular}{|l|l|l|l|}
\hline & Male & Female & Total \\
\hline Total & 13 & 8 & 21 \\
\hline Diabetic & 0 & 1 & 1 \\
\hline Smoker & 5 & 2 & 7 \\
\hline Immunosuppressed & \multicolumn{5}{l|}{} \\
\hline Chemotherapy & 3 & 0 & 3 \\
\hline Corticosteroid & 1 & 0 & 1 \\
\hline
\end{tabular}

Table 2 Demographics and union rates

\begin{tabular}{|l|l|l|}
\hline & Union rate & p-Value \\
\hline Smoker & $4 / 7(57.1 \%)$ & \multirow{2}{*}{$p=0.28$} \\
\hline Nonsmoker & $12 / 14(85 \%)$ & \\
\hline Immunosuppressed & $3 / 4(75 \%)$ & \multirow{2}{*}{$p=1.00$} \\
\cline { 1 - 2 } Nonimmunosuppressed & $12 / 17(70.6 \%)$ & \\
\hline
\end{tabular}

antegrade or retrograde in the defect. Data collected included comorbidities such as diabetes, smoking history, and immune suppression (including chemotherapy and/or radiation treatment), as well as complications related to the surgery and any subsequent surgeries required to achieve union.

The postoperative follow-up clinical evaluations, radiology reports, and radiographs were reviewed to assess for union of the graft. The graft was considered united if radiographic review showed an uninterrupted external bony border between the fibula and recipient bone on at least three cortices as well as an obscured or absent osteotomy line (-Figs. 1 and 2). Clinical notes indicating union of the graft and radiology reports were also reviewed and used to corroborate radiographic findings. Radiographs were identified and reviewed in the Centricity PACS-IW (GE Healthcare IT, Little Chalfont, UK) digital radiography system. Primary union at the proximal and distal junctions with respect to the graft orientation and with respect to the limb was determined. Graft orientation refers to how the graft was placed in the defect, that is, whether it is placed antegrade or retrograde in the defect. Limb orientation refers to the anatomical orientation of the limb with the proximal referring to proximal part of the limb and distal referring to the distal aspect of the limb.

The length of the graft utilized and rate of primary union at the proximal and distal graft-recipient interface were analyzed. Pearson correlation coefficient was calculated to determine if a correlation exists between the length of the vascularized fibula graft used and nonunion of the graftrecipient site interface. The rates of primary union at the proximal and distal ends of the graft with respect to the limb and with respect to the graft were analyzed using chi-square or Fisher exact analysis, with a $p<0.050$ considered statistically significant.

\section{Results}

The length of the graft did not have a significant correlation with the rate of nonunion at either graft/recipient junction (-Figs. 3-6). The correlation coefficients for the rate of union versus length at the proximal limb junction and proximal graft junction were both lower than 0.19 and were not statistically significant (-Figs. 3 and 4 ). The correlation coefficients for the rate of union versus length at the distal limb junction and distal graft junction were both less than 0.30 and were not statistically significant (-Figs. 5 and 6).

Overall, 71.4\% (15/21) achieved complete primary union at an average of 8.7 months (5-22 months). Of the six patients who did not achieve primary complete union (both junction sites united), an additional procedure was successful in achieving union in an additional two patients, resulting in a combined primary and secondary union rate of $81 \%$. One of these patients underwent autologous iliac crest bone grafting to the nonunion site, and with a bone stimulator healed after 4 months. The other patient underwent irrigation and debridement with revision of the hardware, and with use of the bone stimulator healed after 16 months.

Of the four remaining patients who did not achieve complete primary union of the graft, one patient has 


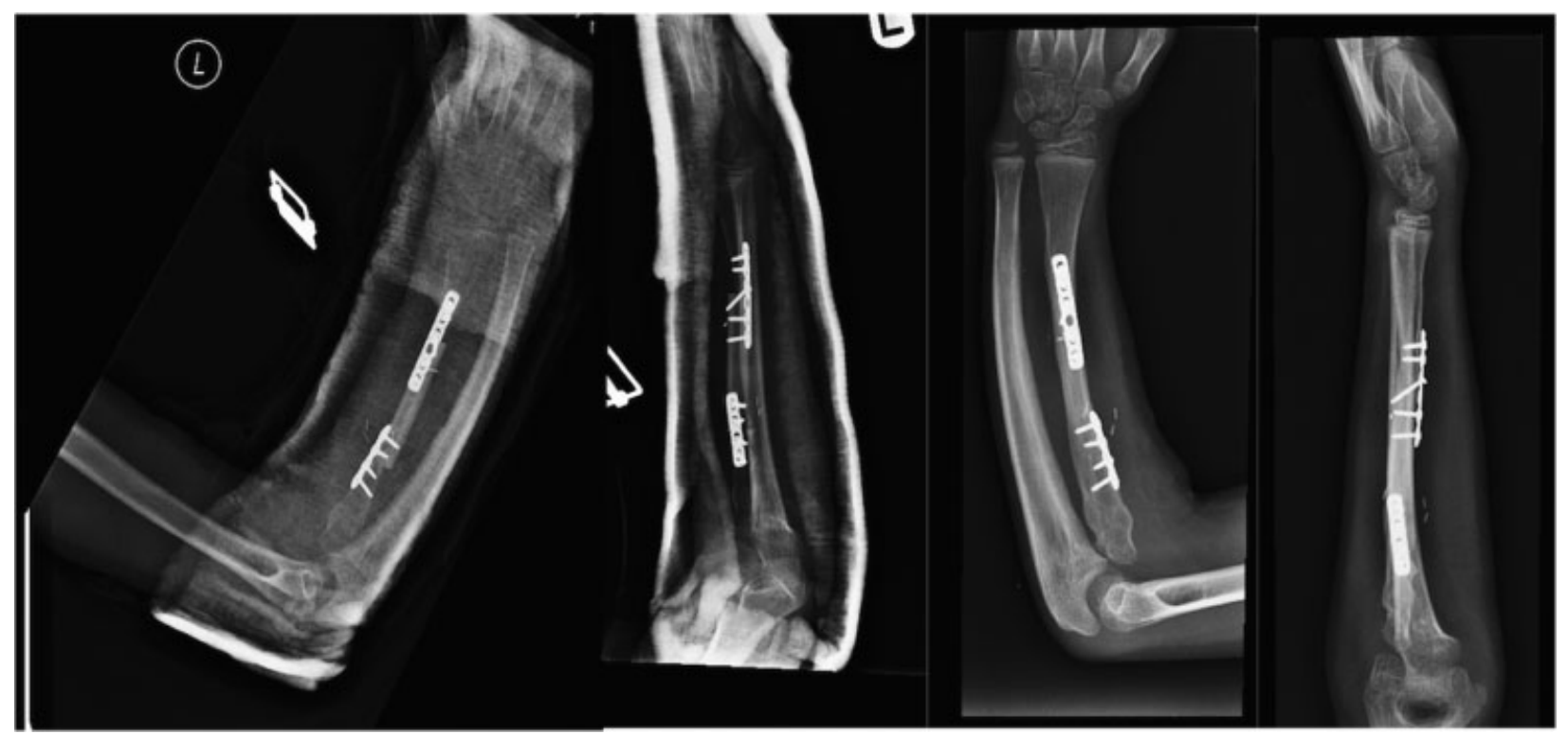

Fig. 1 Radiograph of vascularized fibula grafting to the radius. The images show the immediate postoperative radiographs and final radiographs with complete incorporation with an absent osteotomy line.

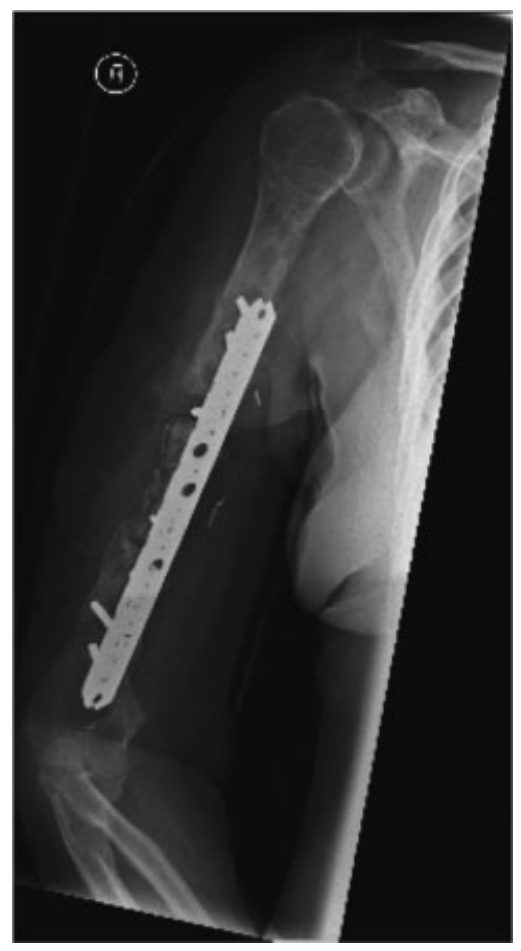

Fig. 2 Radiograph of nonunion of vascularized fibula graft. The image shows the complete absence of bony bridging and clearly seen osteotomy lines representing a complete nonunion of the graft.

undergone multiple irrigation and debridements and currently has an antibiotic spacer in place. Another patient had infection of the graft with removal of the graft and arthrodesis of the elbow with autologous bone graft. The final two patients underwent autologous iliac crest bone grafting and revision of hardware and at last follow-up complete union had not yet occurred.

As stated earlier, a total of six primary nonunions occurred, of which one was a complete nonunion (neither end of the

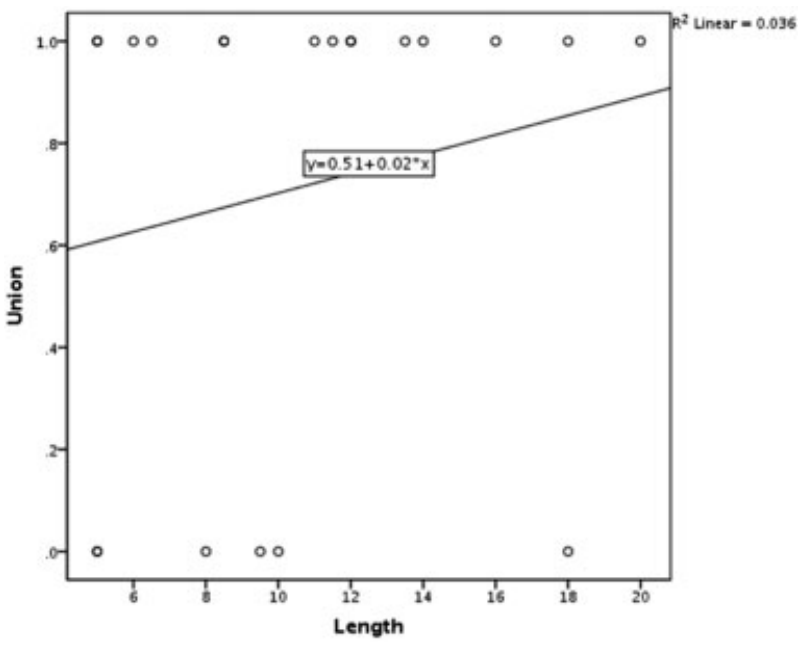

Fig. 3 Union of the proximal junction versus length of graft with respect to the limb. Pearson correlation plot of the rate of union at the proximal junction versus the length of the graft with respect to the limb orientation. Key: 0 , nonunion; 1 , union. Pearson correlation coefficient $0.19(p=0.41)$.

graft united). In the remaining five, one of the graft/recipient junctions united and the other did not. The distal graft/ recipient junction with respect to the limb orientation had a significantly greater rate of primary union versus the proximal graft/recipient junction (20/21 [95.2\%] vs. $15 / 21$ $[71.4 \%]$, respectively; $p=0.038$ ). With respect to the orientation of the graft, there was higher rate of primary union at the distal graft than the proximal graft but this was not statistically significant (19/21 [90.5\%] vs. $16 / 21$ [76.2\%]; $p=0.214$ ).

Vascularized fibula grafting was performed 11 times in the upper extremity and 10 times in the lower extremity (-Table 3). The average length of the graft in the upper extremity was $8.77 \mathrm{~cm}$ and $12.65 \mathrm{~cm}$ in the lower extremity, which was not statistically different $(p=0.054)$. The rate of 


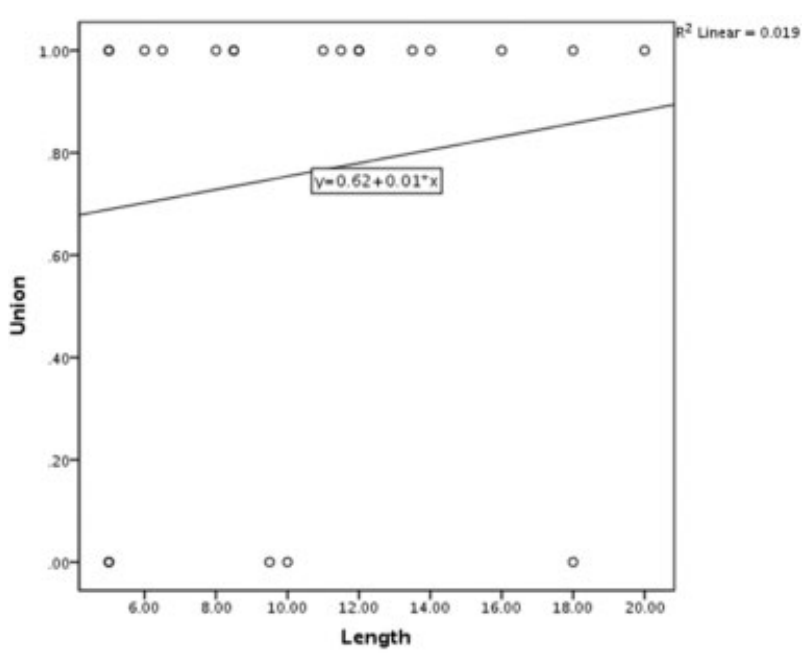

Fig. 4 Union of the proximal junction versus length of graft with respect to the graft. Pearson correlation plot of the rate of union at the proximal junction versus the length of the graft with respect to the graft orientation. Key: 0 , nonunion; 1 , union. Pearson correlation coefficient $0.138(p=0.55)$.

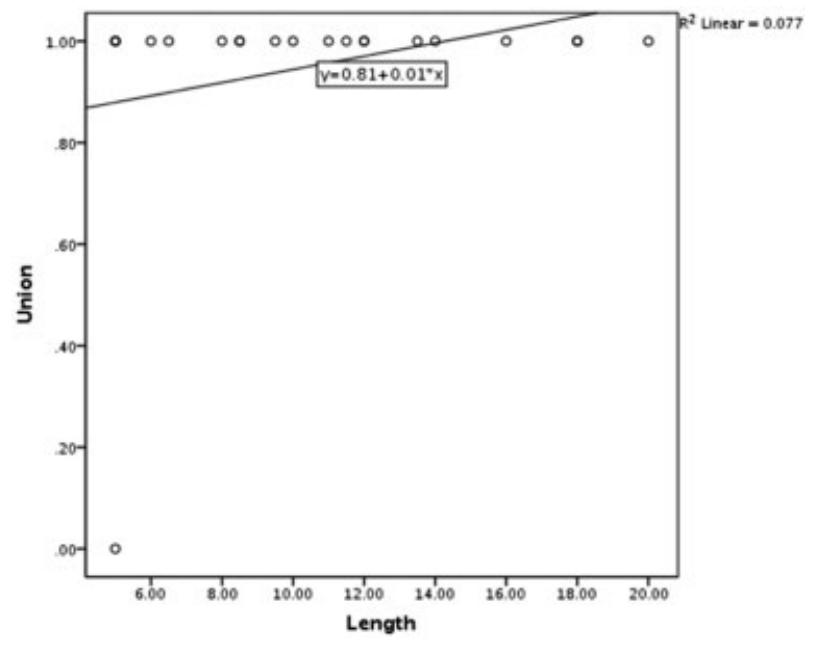

Fig. 5 Union of the distal junction versus length of graft with respect to the limb. Pearson correlation plot of the rate of union at the distal junction versus the length of the graft with respect to the limb orientation. Key: 0 , nonunion; 1 , union. Pearson correlation coefficient $0.277(p=0.22)$.

primary union in the upper versus lower extremities was 54.6 versus $90 \%(p=0.149)$. Rate of combined primary and secondary union was $72.7 \%$ in the upper extremity versus $90 \%$ in the lower extremity $(p=0.586)$. The procedure was performed for tumor resection reconstruction/reconstruc-

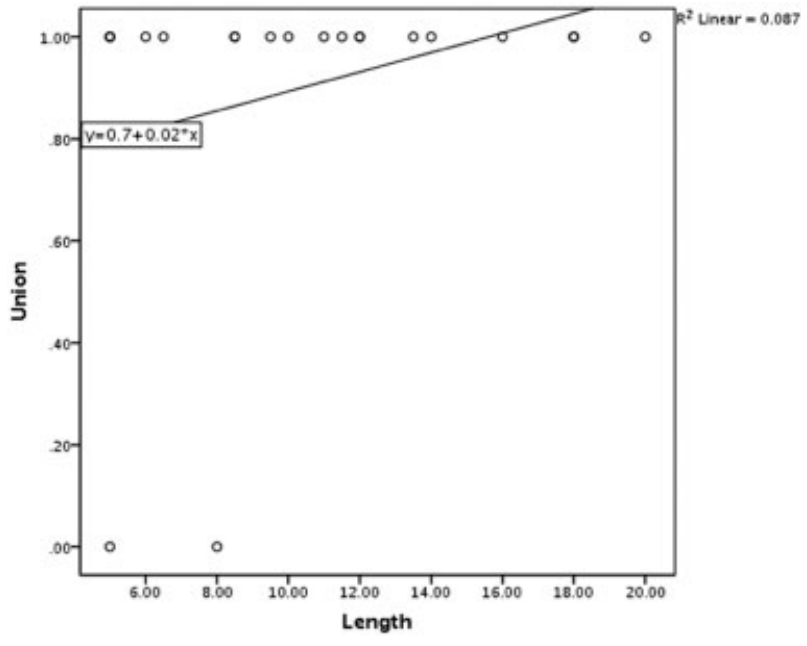

Fig. 6 Union of the distal junction versus length of the graft with respect to the graft. Pearson correlation plot of the rate of union at the distal junction versus the length of the graft with respect to the graft orientation. Key: 0 , nonunion; 1 , union. Pearson correlation coefficient $0.294(p=0.20)$.

tion of nonunions in nine patients, infected nonunions in seven patients, and traumatic nonunions/traumatic bone loss in five patients (-Table 4). The patients with infected nonunions had a primary union rate of $57.1 \%$ (four out of seven united) and an ultimate union rate of $71.4 \%$ (five out of seven) after a secondary procedure, which was not statistically different from the ultimate union rate for the patients who underwent the procedure for tumor resection reconstruction $(p=0.175)$ or traumatic bone loss/nonunion $(p=1.00)$. The primary union rate was different between the tumor resection group and the traumatic nonunion group $(p=0.027)$, and the tumor resection group had a higher overall union rate than the traumatic group although not statistically significant $(p=0.110 ;$ - Table 4).

The surgical technique utilized (i.e., double-barrel vascularized graft vs. single, rigid vs. nonrigid fixation, and antegrade vs. retrograde placement of the graft) did not appear to have a statistically significant effect on union rates ( $\mathbf{- T a b l e ~} \mathbf{5}$ ). A total of five patients had double-barrel vascularized fibula grafting with a union rate of $80 \%(4 / 5$ united), whereas single vascularized fibula grafts had union rate of $68.8 \%$ ( $11 / 16$ united, $p=1.00$ ). In terms of fixation, the graft was stabilized with either rigid fixation, which included compression plating and locked plating, or nonrigid fixation, which included screw, K-wire, or external fixator stabilization. The rigid fixation group had a union

Table 3 Location and indications for vascularized fibula grafting

\begin{tabular}{|l|l|l|l|l|l|l|l|}
\hline Extremity & No. of patients & Tumor & Infection & Trauma & Average length $(\mathbf{c m})$ & Primary union & Overall union \\
\hline Upper & 11 & 3 & 4 & 4 & 8.8 & $6 / 11(54.6 \%)$ & $8 / 11(72.7 \%)$ \\
\hline Lower & 10 & 6 & 3 & 1 & 12.7 & $9 / 10(90 \%)$ & $9 / 10(90 \%)$ \\
\hline & & & & & $p=0.054$ & $p=0.149$ & $p=0.586$ \\
\hline
\end{tabular}

Note: The table shows the number of times vascularized fibula grafting was performed in the upper and lower extremities with the indication for the surgery, average graft length, and union rates achieved in the upper and lower extremities. 
Table 4 Indication for vascularized fibula grafting and union rates

\begin{tabular}{|l|l|l|}
\hline Indication & Primary union & Overall union \\
\hline Tumor & $9 / 9(100 \%)$ & $9 / 9(100 \%)$ \\
\hline Infected nonunion & $4 / 7(57.1 \%)$ & $5 / 7(71.4 \%)$ \\
\hline Traumatic & $2 / 5(40 \%)$ & $3 / 5(60 \%)$ \\
\hline
\end{tabular}

Note: The union rates achieved based on the indication for vascularized fibula grafting. The rate of primary union for the indication for surgery of tumor was significantly higher than for the indication for surgery of traumatic nonunion/bone loss $(p=0.027)$. The rate of primary union was higher for the indication of tumor reconstruction than infection although not statistically different $(p=0.063)$. The rate of primary union was not statistically different between the indications of infection versus trauma $(p=1.00)$. The overall union rate was higher for the indication for surgery of tumor compared with trauma and infection, although not statistically significant ( $p=0.110$ and 0.175 , respectively). There was no significant difference in overall union rate between infection versus trauma $(p=1.00)$.

Table 5 Surgical technique utilized and union rates

\begin{tabular}{|l|l|l|}
\hline Surgical technique & Union rate & $p$-Value \\
\hline Double-barrel graft & $4 / 5(80 \%)$ & $p=1.00$ \\
\cline { 1 - 2 } Single graft & $11 / 16(68.8 \%)$ & \\
\hline $\begin{array}{l}\text { Rigid fixation of } \\
\text { junction sites }\end{array}$ & $16 / 22(72.7 \%)$ & \\
\cline { 1 - 2 } $\begin{array}{l}\text { Nonrigid fixation of } \\
\text { junction sites }\end{array}$ & $19 / 20(95 \%)$ & \\
\cline { 1 - 2 } & $12 / 17(70.6 \%)$ & \\
\cline { 1 - 2 } Retrograde anastomosis & $3 / 4(75 \%)$ & \\
\hline
\end{tabular}

rate of $72.7 \%$ (16/22 junction sites united) compared with the nonrigid fixation group with a union rate of $95 \%$ (19/20 junction sites united; $p=0.096)$. In total, four fibula grafts were anastomosed in a retrograde manner. Those grafts placed retrograde had a $75 \%$ ( $3 / 4$ united) primary union rate compared with $70.6 \%$ (12/17 united) union rate in the grafts placed antegrade $(p=1.00)$.

\section{Discussion}

The length of the fibula graft used did not significantly correlate with nonunion at either junction site. Increased graft length did not correlate with increased nonunion rate. Biomechanical studies looking at the stability at the junction sites of the vascularized fibula graft utilizing different fixation techniques have suggested that as lengths increase there is increase motion and stresses at the junction sites. ${ }^{7,8}$ This could lead to decreased union. One study based on biomechanical data suggested that single vascularized fibula graft should not exceed $15 \mathrm{~cm}^{7}$ Another biomechanical study suggested graft length should not exceed $12 \mathrm{~cm} .{ }^{8}$ However, clinical studies that have included information on length of graft and overall union have not found increased overall nonunion rates with longer grafts. ${ }^{9}$ Furthermore, the length of the graft has not been found to correlate with other parameters of graft incorporation or healing such as graft hypertrophy. ${ }^{10,11}$ Increasing graft length has been associated with increased rates of graft fracture, ${ }^{3}$ although no cases of fracture were seen in the present study. Increased graft length does not seem to result in lower union rates but may increase risk of complication.
Vascularized fibula grafting is a useful tool in the treatment of bone defects. The primary union rate in this study was $71.4 \%$ at an average of 8.7 months. Other series have reported union rates ranging from 41 to $100 \%{ }^{3-6,9,11-16}$ Our results are in the middle of previously reported rates. In all but one patient, primary union occurred at least at one end of the graft, suggesting that when a nonunion occurs it is likely to be only at one of the junctions and not both. A study looking at the results of vascularized fibular graft for defects of the upper extremity has shown similar findings, reporting that 37 out of 40 junction sites healed. ${ }^{17}$

To our surprise, we found that the proximal graft/recipient junction was more likely to go on to nonunion than the distal junction. A statistically significant worse primary union rate occurred at the proximal graft/recipient junction with respect to the limb orientation. This has not been previously reported in the literature. A study by Minami et al reported that 13 of the patients in their series achieved primary union at one end of the fibula and secondary at the other, but does not state at which end secondary union occurred. ${ }^{3} \mathrm{~A}$ previous study by de Boer et al found no difference in the rate of union at the proximal and distal end of the transferred fibula, but that study looked at overall union, not just primary union. ${ }^{9}$ However, the same study found improved union rates at the proximal graft/recipient junction with use of stable internal fixation, which was not seen at the distal junction. ${ }^{9}$ They also saw improved proximal union rates with the use of bone graft, which was not found to improve union rates at the distal junction. ${ }^{9}$ These findings support the notion that the proximal junction has a slower or more difficult time healing. 
Given these findings, one would expect that if a nonunion were to occur it would occur at the proximal junction.

It is unclear why the proximal junction would have a higher rate of nonunion, but mechanical factors and vascularity of the fibula could play a role. de Boer et al postulated that the proximal graft junction would be slower to heal and unite than the distal because the proximal is typically located toward the diaphysis and the distal graft junction located toward the metaphsysis. ${ }^{9}$ Faster fracture healing and faster union is expected in metaphyseal bone. However, in the present study the proximal end is not overwhelmingly toward the diaphysis.

Another possible contribution to the slower union proximally could be related to the vascularity of the fibula. The fibula has both an endosteal and periosteal blood supply from branches off of the peroneal artery. The endosteal blood supply comes from the nutrient artery, which enters the fibula at the junction of the proximal one-third and distal two-thirds of the diaphysis and then gives rise to ascending and descending branches. The periosteal blood supply comes from eight to nine periosteal branches, which are mostly in the middle third of the diaphysis. The fibular head and epiphysis blood supply comes from a branch off of the anterior tibial artery. Perhaps, the more proximal portion of the fibula grafts represents more of a watershed area in the vascularity, which may contribute to slower healing at the proximal junction when used for nonunion repairs.

In the present study, the primary rates of union were higher in the lower extremity than in the upper extremity, although not statistically significant. This is somewhat surprising considering the length of the grafts utilized were on average shorter in the upper extremity. In another study looking at vascularized fibula grafting, the upper extremity reconstructions had a trend toward improved overall union rates. ${ }^{9}$ In our study, there were more infected and traumatic bone loss and less tumor resection reconstruction as the indication for vascularized fibula grafting in the upper extremity than the lower extremity. In this series, those patients with severe open wounds and bone loss from trauma and infected nonunions had worse primary union rates than reconstruction of tumor resections. Other studies have shown worse rates of union in infected nonunions and osteomyelitis than tumor reconstructions. ${ }^{2,9}$ This could account for the lower rates of primary union in the upper extremity compared with the lower.

There were weaknesses in the present study, namely, the retrospective nature of the study and the small sample size. Being retrospective in nature, it is difficult to control for all potential confounders when assessing rates of union of the graft and the role the length of the graft may play. The sample size was small consisting of only 21 included patients, which limits the conclusions that can be made and the extent of analysis made. However, this is a relatively uncommon procedure and the size of the study is average in terms of the reported studies in the literature. A larger sample size may have produced a more significant difference in the rates of union between the proximal and distal junction sites of the graft and further subanalysis of factors contributing to nonunion.

\section{Conclusion}

In summary, vascularized fibula grafting is a useful tool in the reconstruction of large bony defects with good rates of union. The length of the graft does not appear to have an association with the rate of primary union at either graft/recipient junction. Primary union reliably occurs at least at one end and complete nonunion of the graft is rare. If primary union is not achieved, likely the nonunion will have occurred at the proximal graft/ recipient junction with respect to the limb orientation.

\section{Acknowledgment}

The authors thank Linda Chen, MS, for assistance with statistical analysis.

\section{References}

1 Taylor GI, Miller GD, Ham FJ. The free vascularized bone graft. A clinical extension of microvascular techniques. Plast Reconstr Surg 1975;55(5):533-544

2 Han CS, Wood MB, Bishop AT, Cooney WP III. Vascularized bone transfer. J Bone Joint Surg Am 1992;74(10):1441-1449

3 Minami A, Kasashima T, Iwasaki N, Kato H, Kaneda K. Vascularised fibular grafts. An experience of 102 patients. J Bone Joint Surg $\mathrm{Br}$ 2000;82(7):1022-1025

4 Duffy GP, Wood MB, Rock MG, Sim FH. Vascularized free fibular transfer combined with autografting for the management of fracture nonunions associated with radiation therapy. J Bone Joint Surg Am 2000;82(4):544-554

5 González del Pino J, Bartolomé del Valle E, Graña GL, Villanova JF. Free vascularized fibular grafts have a high union rate in atrophic nonunions. Clin Orthop Relat Res 2004;(419):38-45

6 Yajima H, Kobata Y, Shigematsu K, et al. Vascularized fibular grafting in the treatment of methicillin-resistant Staphylococcus aureus osteomyelitis and infected nonunion. J Reconstr Microsurg 2004;20(1):13-20

7 Chen H, Zhang Y, Xia H, Wang F, Li Z, Chen X. Stability of tibial defect reconstruction with fibular graft and unilateral external fixation: a finite element study. Int J Clin Exp Med 2014;7(1): 76-83

8 Ma L, Zhou Y, Zhang Y, et al. Biomechanical evaluation with finite element analysis of the reconstruction of femoral tumor defects by using a double-barrel free vascularized fibular graft combined with a locking plate. Int J Clin Exp Med 2014;7(9): 2425-2434

9 de Boer HH, Wood MB, Hermans J. Reconstruction of large skeletal defects by vascularized fibula transfer. Factors that influenced the outcome of union in 62 cases. Int Orthop 1990;14(2):121-128

10 Gao YS, Ai ZS, Yu XW, et al. Free vascularised fibular grafting combined with a locking plate for massive bone defects in the lower limbs: a retrospective analysis of fibular hypertrophy in 18 cases. Injury 2012;43(7):1090-1095

11 Kovoor CC, Jayakumar R, George V, Padmanabhan V, Guild A, Viswanath $S$. Vascularized fibular graft in infected tibial bone loss. Indian J Orthop 2011;45(4):330-335

12 Chhabra AB, Golish SR, Pannunzio ME, Butler TE Jr, Bolano LE, Pederson WC. Treatment of chronic nonunions of the humerus with free vascularized fibula transfer: a report of thirteen cases. J Reconstr Microsurg 2009;25(2):117-124

13 Khira YM, Badawy HA. Pedicled vascularized fibular graft with Ilizarov external fixator for reconstructing a large bone defect of the tibia after tumor resection. J Orthop Traumatol 2013;14(2): 91-100 
14 Lee KS, Han SB, Baek JR. Free vascularized osteocutaneous fibular graft to the tibia in 51 consecutive cases. J Reconstr Microsurg 2004;20(4):277-284

15 Pollock R, Stalley P, Lee K, Pennington D. Free vascularized fibula grafts in limb-salvage surgery. J Reconstr Microsurg 2005;21(2): 79-84
16 Tanaka K, Maehara H, Kanaya F. Vascularized fibular graft for bone defects after wide resection of musculoskeletal tumors. J Orthop Sci 2012;17(2):156-162

17 Soucacos PN, Korompilias AV, Vekris MD, Zoubos A, Beris AE. The free vascularized fibular graft for bridging large skeletal defects of the upper extremity. Microsurgery 2011;31(3):190-197 